\title{
DESCRIPCIÓN DE LA LARVA DE PHYLLOPHAGA (PHYTALUS) RUFOTESTACEA (MOSER) (COLEOPTERA: MELOLONTHIDAE) EN CHIAPAS, MÉXICO
}

\author{
Concepción RaMírez-SALINAS1, Miguel-Ángel MoRóN22, \\ Adriana E. CASTRo-Ramírez ${ }^{1}$ y Cutberto PACheCo-Flores 1 \\ ${ }^{1}$ El Colegio de La Frontera Sur (ECOSUR), Apdo. Postal 63, San Cristóbal de Las Casas, Chiapas, \\ 29290 MÉXICO.cramirez@sclc.ecosur.mx \\ 2Instituto de Ecología, A.C. Apdo. Postal 63 Xalapa, Veracruz 91000, MÉXICO. \\ miguel.moron@inecol.edu.mx
}

Ramírez-Salinas, C., M. A. Morón, A. E. Castro-Ramírez y C. Pacheco-Flores. 2009. Descripción de la larva de Phyllophaga (Phytalus) rufotestacea (Moser) (Coleoptera: Melolonthidae) en Chiapas, México. Acta Zool. Mex. (n. s.) 25(1):1-8.

RESUMEN. Se describe la larva de tercer estadio de Phyllophaga (Phytalus) rufotestacea (Moser, 1918) con base en ejemplares criados a partir de huevos obtenidos de adultos en cautiverio recolectados en San Cristóbal de Las Casas, Chiapas, México. Se incluyen datos sobre sus hábitos y ciclo de vida. Palabras Clave: Morfología, taxonomía, gallina ciega, edafícolas, saprófagas.

Ramírez-Salinas, C., M. A. Morón, A. E. Castro-Ramírez \& C. Pacheco-Flores. 2009. Description of the larva of Phyllophaga (Phytalus) rufotestacea (Moser) from Chiapas, Mexico. Acta Zool. Mex. (n. s.) $25(1): 1-8$.

ABSTRACT. The third-instar larva of Phyllophaga (Phytalus) rufotestacea (Moser, 1918) is described and illustrated, based on specimens reared from eggs laid by captive adults collected in San Cristobal de Las Casas, Chiapas, Mexico. Some data on the life cycle and habits are included.

Key Words: Morphology, taxonomy, white grubs, edaphicolous, saprophagous.

\section{INTRODUCCIÓN}

Es necesario conocer las características morfológicas y los hábitos de las larvas de los coleópteros Scarabaeoidea o Lamelicornios, conocidas comúnmente como "gallina ciega" o "kolom", para lograr un control adecuado de las larvas de las especies nocivas para los cultivos agrícolas, y evitar el daño a las especies benéficas que habitan en los suelos cultivados. Algunas especies de Melolonthidae en el estado adulto son fitófagas o saprófagas, mientras que sus larvas se alimentan con raíces; en tanto que los adultos de otras especies consumen el follaje de diversas plantas y sus larvas son saprófagas (Ritcher 1958, Morón 1983, 1994, Morón et al.1996). En lo que a larvas de Melolonthidae se refiere, a la fecha sólo se conoce el $1 \%$ de la diversidad de especies que habitan en los suelos cultivados de América Latina (Ramírez et al. 2000; 2004). 
Phyllophaga (Phytalus) rufotestacea (Moser) se describió originalmente con ejemplares colectados en la ciudad de Guatemala (Moser 1918; Cano y Morón 1998), y se registró por primera vez en San Cristóbal de Las Casas, Chiapas, México por Pacheco-Flores y Castro Ramírez (2005). Las larvas de P. rufotestacea probablemente se desarrollan en el suelo del arbolado o en pastizales, pues a pesar de los numerosos muestreos intensivos en la región no se les ha encontrado en terrenos cultivados (Pacheco-Flores y Castro-Ramírez 2005).

Los objetivos del presente trabajo consisten en describir por primera vez las larvas de tercer estadio de Phyllophaga (Phytalus) rufotestacea, y compararlas con las larvas de otras especies del género.

\section{MATERIAL Y MÉTODOS}

Los adultos se recolectaron el 9 y 25 de mayo de 2005, en la parte sur de la ciudad de San Cristóbal de Las Casas, Chiapas. En el laboratorio de bioensayos de El Colegio de La Frontera Sur (ECOSUR) para obtener huevos se colocaron 37 hembras y 14 machos en recipientes plásticos con suelo húmedo, cubiertos con una tela fina de organza; se les alimentó con hojas de frijol bótil (Phaseolus coccineus) y sus larvas con raíces de "chisme" Sedum praealtum parvifolium R. T. Clausen (Crassulaceae). Cuando las larvas alcanzaron el tercer estadio de su desarrollo se fijaron durante 10 días en solución de Pampel (Morón y Terrón 1988) y se conservaron en alcohol etílico al $70 \%$. Con cinco larvas de tercer estadio fijadas se obtuvieron las dimensiones de la cápsula cefálica. Las descripciones e ilustraciones se efectuaron con el apoyo de un estereomicroscopio Carl Zeiss Stemi SV6 provisto con cámara clara. Los caracteres considerados en las descripciones y la terminología utilizada, son los propuestos por Böving (1942), Ritcher (1966) y Morón (1986). Los ejemplares estudiados están depositados en las colecciones entomológicas de ECOSUR, San Cristóbal de Las Casas, Chiapas, y del Instituto de Ecología, A.C. Xalapa, Veracruz.

\section{Phyllophaga (Phytalus) rufotestacea (Moser, 1918)}

Larva de tercer estadio. Cabeza. Anchura máxima de la cápsula cefálica: 3.41 a $3.75 \mathrm{~mm}$. $(\mathrm{N}=5)$. Superficie del cranium finamente rugosa, parda amarillenta. Frente con 1 ó 2 sedas frontales exteriores y 1 seda frontal posterior a cada lado; y 14-17 sedas frontales anteriores; con dos sedas en los ángulos anteriores de la frente; el resto de la superficie craneal con 2 ó 3 sedas dorso-epicraneales, con 1 seda epicraneal o sin ella, y 6-7 sedas paraocelares a cada lado (Fig. 1). Clípeo con 2 sedas centrales y 3-6 sedas laterales a cada lado. Labro asimétrico, con los márgenes laterales redondeados, 10-15 sedas posteriores, 2 sedas centrales y 3 sedas laterales a cada lado (Fig. 1). La epifaringe tiene una anchura de 1.54 a $1.56 \mathrm{~mm}$ y una longitud de 1.25 a $1.37 \mathrm{~mm}$, sin zygum y con epizygum alargado, irregular, pardo 
obscuro; haptomerum moderadamente prominente con 6 a 7 heli; cada plegmatium formado con 9-11 plegmata anchos. Proplegmatia con 17-19 plegmata muy finos y cercanos entre sí. Dexiophoba irregular y fragmentada y laeophoba grande, extendida a través del borde de la laeotorma. Dexiotorma casi recta; laeotorma corta; apotorma estrecha y aguzada. Placa esclerosada reducida, estrecha, angulosa. Crepis muy estrecho, bien definido. Cada acanthoparia con 9-11 sedas espiniformes recurvadas. Chaetoparia moderadamente desarrollada con numerosas sensilas en el límite de la gymnoparia (Fig. 4). Mandíbulas sin área estriduladora ventral. Área incisiva de la mandíbula derecha con una lámina cortante distal separada por una escotadura del dientecillo incisivo proximal; márgenes internos sin dientes (Fig. 3). Área incisiva de la mandíbula izquierda con una lámina cortante distal poco pronunciada; área molar de la mandíbula izquierda con el lóbulo distal (M1) bien desarrollado (Fig. 2); brustia moderadamente setosa; área molar de la mandíbula derecha con dos rebordes diferenciados; calx prominente y brustia moderadamente setosa. Maxilas con un uncus en la galea y tres unci separados basalmente y alineados en la lacinia, y con hileras de 4-5 heli a cada lado (Figs. 5, 7). Área estriduladora maxilar con 9-15 dientecillos (Figs. 6-7). Escleroma hipofaríngeo asimétrico, con el lado derecho prominente (Fig. 8). Último artejo antenal con un área sensorial ovalada en la superficie dorsal (Fig. 9) y dos áreas sensoriales en la superficie ventral.

Tórax. Los estigmas respiratorios miden $0.2-0.22 \mathrm{~mm}$ de largo por $0.28-0.3 \mathrm{~mm}$ de ancho; placa respiratoria amarilla rojiza, uniformemente recurvada en forma de "C" (Fig. 10). Pronoto con un escleroma lateral alargado a cada lado, amarillo cremoso, poco contrastante, con sedas largas y medianas. Proprescudo con 25-27 sedas cortas y largas irregularmente distribuidas; proescudo con 9-13 sedas cortas y largas irregularmente situadas; mesoprescudo con 9-10 sedas cortas y largas; mesoescudo con 8-19 sedas cortas y largas; mesoescutelo con 7-10 sedas cortas y largas; metaprescudo con 11-15 sedas cortas y largas; metaescudo con 14-17 seda cortas y largas; metaescutelo con 5-9 sedas cortas y largas. Uñas tarsales pro y mesotorácicas similares, alargadas, agudas, con dos sedas cercanas a su base (Figs 12-13.); uñas metatorácicas reducidas, con dos sedas largas, con la parte terminal de la pata más grande que las anteriores (Fig. 15).

Abdomen. Estigmas de los segmentos abdominales I a VIII semejantes en diámetro, de $0.1-0.22 \mathrm{~mm}$ de largo por 0.12 a $0.28 \mathrm{~mm}$ de ancho. Placa respiratoria amarillo rojiza, regularmente recurvada en forma de "C" (Fig. 11). Dorsa de los segmentos abdominales de I-VI con numerosas sedas espiniformes y algunas sedas largas distribuidas en sentido transversal; segmentos abdominales VII-IX sin sedas espiniformes, con hileras de sedas largas situadas transversalmente. Venter de los segmentos abdominales I-IX con 4-22 sedas largas en hileras transversales. Raster (Fig. 14) con 6-8 pali cortos, gruesos, ligeramente recurvados, no organizados en palidia; septula no definida; tegilla con 21 a 29 sedas hamate cortas; campus con 9 a 
12 sedas largas y finas; barbula ligeramente setosa y amarillenta. Labio anal dorsal con pocas sedas espiniformes y sedas largas finas. Labio anal ventral claramente escotado. Abertura anal en forma de "Y". Longitud dorsal aproximada del cuerpo de 2.1 a $2.5 \mathrm{~cm}$. El cuerpo de la larva es blanco hialino y moderadamente robusto.

Material examinado: cinco larvas de tercer estadio, criadas en México: Estado de Chiapas, municipio de San Cristóbal de Las Casas, $2130 \mathrm{~m}$ altitud, obtenidas de huevos depositados por hembras en cautiverio, colectadas el 25-V-2006, que alcanzaron el tercer estadio entre el 28 y el 30 -IX-2006.

Biología. Los adultos fueron recolectados el 25 de mayo y 9 de junio de 2005 en una parcela agrícola sobre plantas de frijol ( $P$. coccineus) y arvenses, entre las 19.00 y 21:30 hrs. (horario de verano), las hembras ovipositaron de 14 a 21 días después de haber sido capturadas. Cada una depositó entre 2 y 9 huevos, el periodo de incubación de éstos fue de 9 a 28 días, que abarcaron del 9 de junio al 6 julio. Las larvas de primer estadio se desarrollaron en un lapso de 23 a 37 días (7 de julio al 14 de agosto); las de segundo estadio se desarrollaron durante 26 a 67 días (2 de agosto al 9 de octubre), mientras que las de tercer estadio se observaron del 7 de septiembre al 13 de diciembre, sin embargo todas murieron sin llegar a pupar. Durante su desarrollo en cautiverio las larvas se alimentaron con raíces de $S$. praealtum parvifolium desde el primer estadio, pero durante octubre y noviembre fueron más voraces. En diciembre las larvas tuvieron más movimiento y consumieron también el suelo. La larva de $P$. rufotestacea se desprende de la exuvia anterior por la parte terminal del abdomen, al mudar de un estadio a otro la cabeza y las patas son completamente blancas y en menos de 5 horas adquieren el color amarillento. Los adultos de $P$. rufotestacea vuelan del 9 de junio al 8 de septiembre y son atacados por el endoparasitoide Cryptomeigenia sp. (Diptera: Tachinidae) (Ramírez-Salinas et al. 2006). Es posible que las hembras hayan depositado pocos huevos debido a su rápida mortalidad causada por dichos parasitoides.

Comentarios taxonómicos. Esta es la primera descripción de una larva de tercer estadio del género Phyllophaga que no presenta los pali organizados claramente como palidia, lo cuál inmediatamente la distingue de las larvas de otras especies de Phyllophaga descritas hasta el momento. Como características adicionales presenta 2 sedas en los ángulos anteriores de la frente, una seda epicraneal, clípeo con 3 a 6 sedas laterales a cada lado, y tiene proplegmatia larga y ensanchada. Comparativamente, las larvas de seis especies de Phyllophaga, descritas para Amatenango del Valle, Chiapas, tienen palidia bien diferenciadas en hileras longitudinales, presentan una seda en los ángulos anteriores de la frente, 2 a 4 sedas epicraneales; clípeo con 2 sedas laterales a cada lado; y sólo $P$. tumulosa y $P$. ravida tienen proplegmatia (Ramírez-Salinas et al. 2000). 

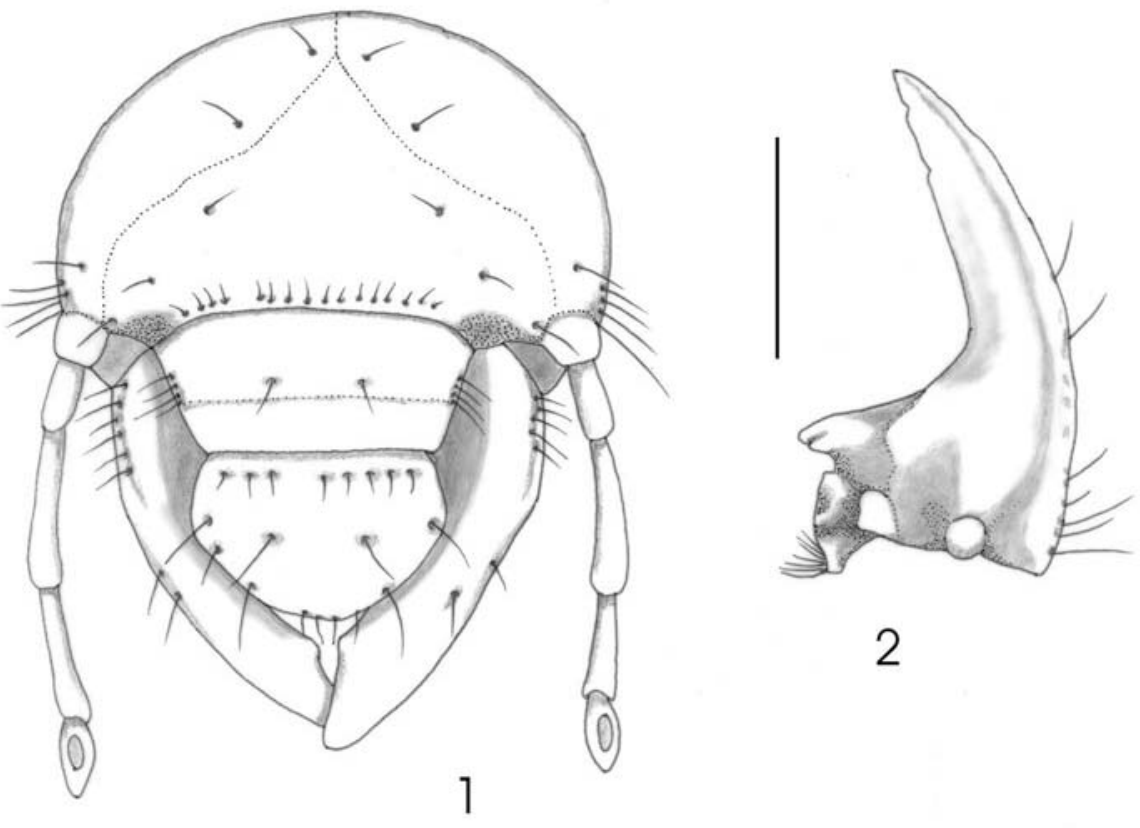

2
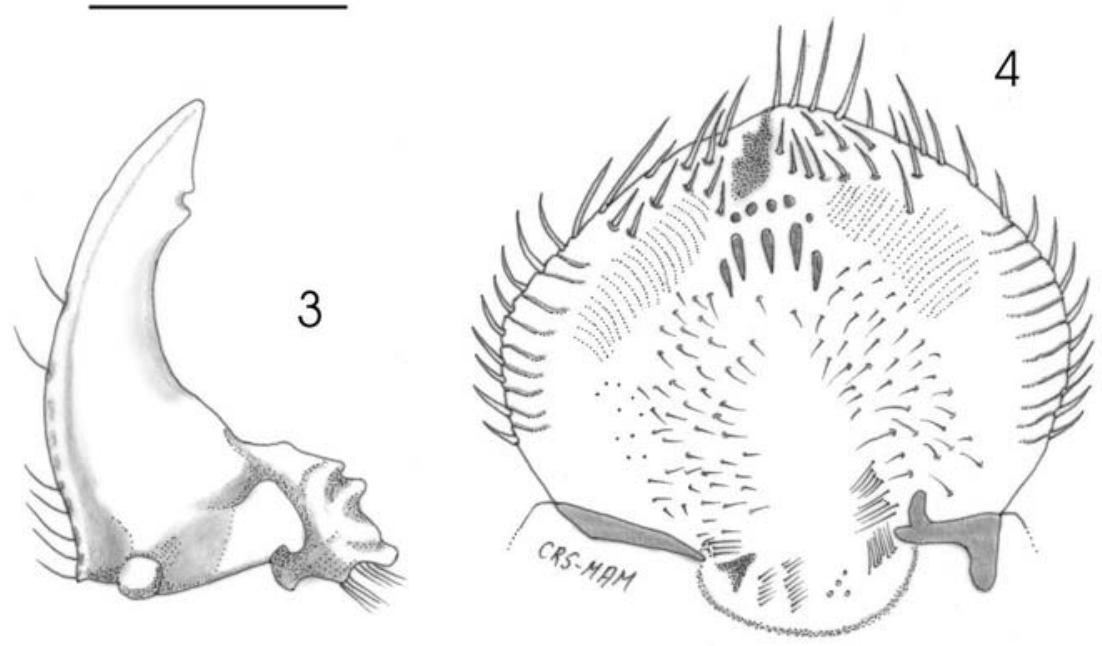

Figuras 1- 4. Estructuras diagnósticas de la larva de tercer estadio de Phyllophaga rufotestacea. 1) Cápsula cefálica, vista frontal. 2) Mandíbula izquierda, vista ventral. 3) Mandíbula derecha, vista ventral. 4) Epifaringe. Líneas de escala $=1 \mathrm{~mm}$. 

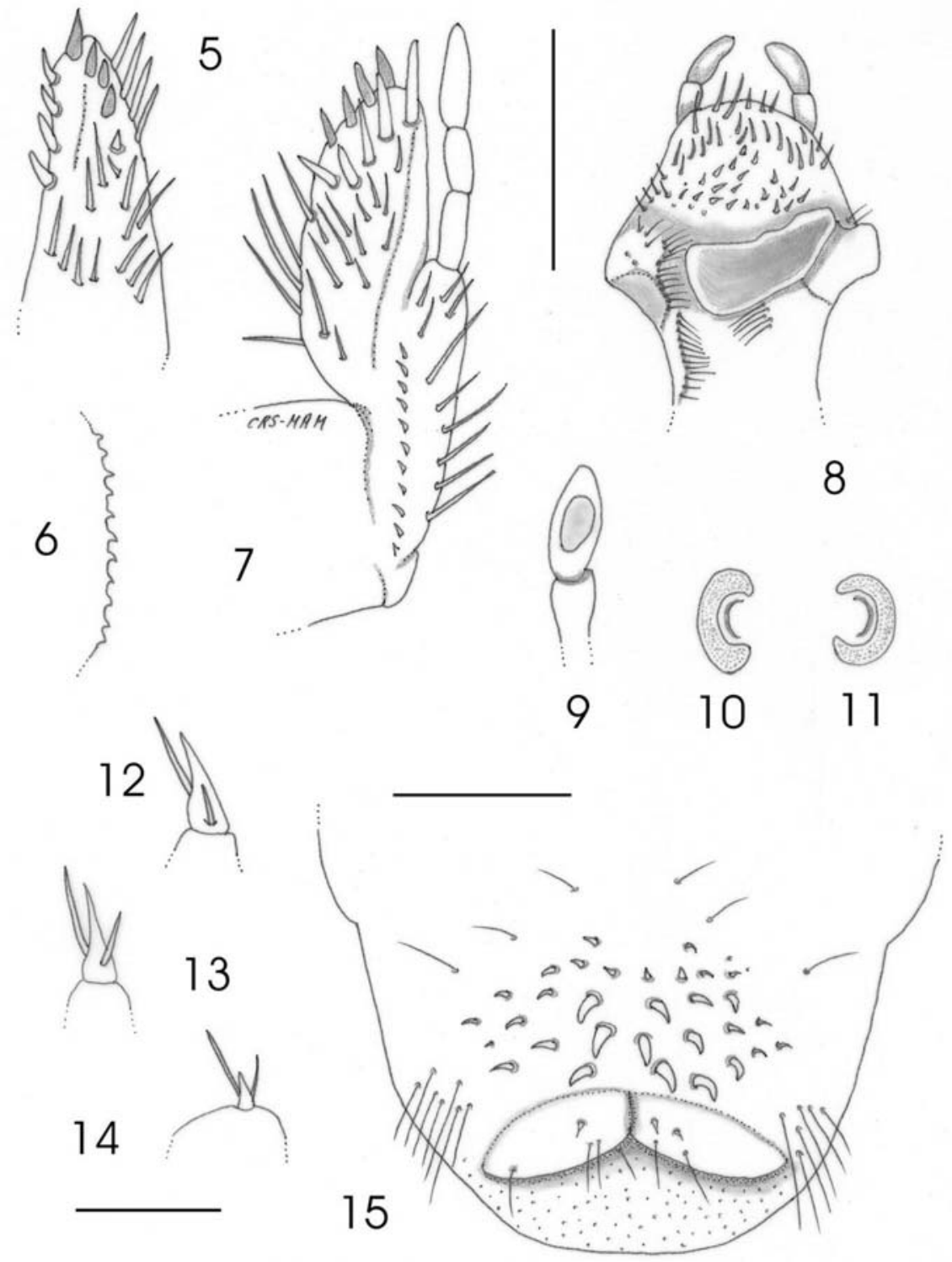

Figuras 5-15. Estructuras diagnósticas de la larva de tercer estadio de Phyllophaga rufotestacea. 5) Ápice de la mala derecha, vista mesial. 6) Detalle de los dientecillos estriduladores maxilares. 7) Maxila derecha, vista dorsal. 8) Labio hipofaringe, vista dorsal. 9) Aspecto dorsal del último artejo antenal. 10) Estigma protorácico izquierdo. 11) Estigma izquierdo del $4^{\circ}$ segmento abdominal. 12) Uña protarsal. 13) Uña mesotarsal. 14) Uña metatarsal. 15) Raster. Líneas de escala figuras 5-8, $15=$ $1 \mathrm{~mm}$; figuras 9-14 $=0.5 \mathrm{~mm}$. 


\section{Clave para identificar larvas de Phyllophaga comunes en los suelos de los Altos de Chiapas (modificada de Ramírez-Salinas et al. 2004)}

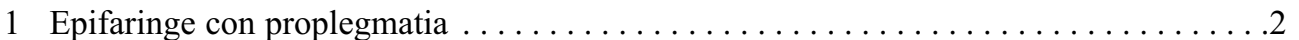

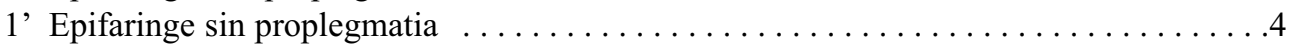

2 Con 6-8 pali cortos, gruesos y recurvados, con sus ápice dirigidos principalmente hacia atrás, distribuidos en la región central posterior del raster. Anchura de la cápsula cefálica $3.4-3.7 \mathrm{~mm}$.......................... (Phytalus) rufotestacea (Moser)

2' Con 16 a 42 pali cortos, con sus ápices dirigidos hacia la línea media, organizados en 2 palidia longitudinales paralelas o convergentes, separadas por una septula bien definida ......... 3

3 Anchura de la cápsula cefálica 3.8-5.1 mm. Palidia casi paralelos, cada palidium formado por 14-21 pali cortos. Anchura de la cápsula cefálica $4.5-5.1 \mathrm{~mm} \ldots \ldots \ldots \ldots \ldots$. $\ldots \ldots \ldots \ldots \ldots \ldots \ldots \ldots \ldots \ldots \ldots \ldots \ldots$. (Phyllophaga) ravida (Blanchard)

3' Extremos posteriores de las palidia ligeramente convergentes, cada palidium formado por 8-15 pali cortos. Anchura de la cápsula cefálica $3.8-4.1 \mathrm{~mm} \ldots \ldots \ldots \ldots \ldots \ldots \ldots$ . . . . . . . . . . . . . . . . . . . . . . . . . . . (Chlaenobia) tumulosa (Bates)

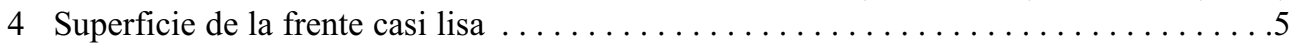

4' Superficie de la frente finamente rugosa $\ldots \ldots \ldots \ldots \ldots \ldots \ldots \ldots \ldots \ldots \ldots \ldots \ldots \ldots$

5 Palidia ligeramente recurvadas, convergentes en ambos extremos, cada palidium formado por 15-27 pali cortos. Anchura de la cápsula cefálica 4.1-4.8 mm . . . . . . . . . . . $\ldots \ldots \ldots \ldots \ldots \ldots \ldots \ldots \ldots \ldots \ldots \ldots \ldots \ldots \ldots$ (Phytalus) obsoleta (Blanchard)

5' Palidia casi paralelas, cada palidium formado por 23-30 pali cortos. Anchura de la cápsula cefálica 5.6-6.2 mm .................. (Phyllophaga) menetriesi (Blanchard)

6 Palidia muy recurvadas, cercanamente convergentes en ambos extremos, delimitando una septula ovalada; cada palidium formado por 19-25 pali cortos. Anchura de la cápsula cefálica 4.9-5.2 mm ................ (Phyllophaga) testaceipennis (Blanchard)

6' Ambos extremos de las palidia ligeramente convergentes, que delimitan una septula alargada; cada palidium formado por 11-18 pali cortos. Anchura de la cápsula cefálica 6.1$6.7 \mathrm{~mm} . \quad \ldots \ldots \ldots \ldots \ldots \ldots \ldots \ldots \ldots \ldots \ldots \ldots \ldots \ldots \ldots$ (Phyllophaga) tenuipilis (Bates)

AGRADECIMIENTOS. Al Dr. Fernando Correa por facilitarnos el acceso a su propiedad para llevar a cabo el trabajo de campo. Esta publicación es una contribución a la línea de investigación "Coleópteros Lamelicornios de América Latina" del Instituto de Ecología, A.C. (cuenta 2000910011).

\section{LITERATURA CITADA}

Cano, E.B. \& M.A. Morón. 1998. Las especies de Phyllophaga (Coleoptera: Scarabaeidae: Melolonthinae) de Guatemala. Diversidad, Distribución e importancia. Pp: 7-18. In: M.A. Morón. \& A. Aragón (Eds.). Avances en el estudio de la diversidad, importancia y manejo de los coleópteros edaficolas americanos. Publicación especial de la Benemérita Universidad Autónoma de Puebla y la Sociedad Mexicana de Entomología, A.C. Puebla, México.

Böving, A. 1942. A classification of larvae and adults of the genus Phyllophaga (Coleoptera: Scarabaeidae). Memoirs of the Entomological Society of Washington 2: 1-95.

Morón, M.A. 1983. Introducción a la biosistemática y ecología de los coleópteros Melolonthidae edafícolas de México. Pp. 1-14. In: II Mesa Redonda sobre Plagas del Suelo. Sociedad Mexicana de 
Entomología. Chapingo, México.

Morón, M.A. 1986. El género Phyllophaga en México. Morfología, distribución y sistemática supraespecifica (Insecta: Coleoptera). Publicación 20. Instituto de Ecología. México.

Morón, M.A. 1994. Aspectos biológicos sobre Scarabaeidae (sensu lato) (Insecta: Coleoptera). Pp.151158. In: Memorias XXI Congreso Sociedad Colombiana de Entomología. SOCOLEN. Medellín, Colombia.

Morón, M.A., S. Hernández-Rodríguez \& A. Ramírez. 1996. El complejo "gallina ciega" (Coleoptera: Melolonthidae) asociado a la caña de azúcar en Tepic, Nayarit México. Folia Entomológica Mexicana 98: 1-44.

Morón, M.A. \& R. Terrón. 1988. Entomología Práctica. Una guía para el estudio de los insectos con importancia agropecuaria, médica, forestal y ecológica de México. Publicación 22. Instituto de Ecología, A. C. México, D. F.

Pacheco-Flores, C. \& A.E. Castro-Ramírez. 2005. Primer registro de Phyllophaga (Phytalus) rufotestacea (Moser, 1918) (Coleoptera: Scarabaeoidea) para México y Chiapas. Acta Zoológica. Mexicana (n.s.) 21(2): 157-158.

Ramírez-Salinas, C., M.A. Morón \& A.E. Castro-Ramírez. 2000. Descripción de los estados inmaduros de seis especies de Phyllophaga (Coleoptera: Melolonthidae; Melolonthinae) de la región Altos de Chiapas, México. Folia Entomológica Mexicana 109: 73-106.

Ramírez-Salinas, C., M.A. Morón \& A.E. Castro-Ramírez. 2004. Descripción de los estados inmaduros de tres especies de Anomala, Ancognatha y Ligyrus (Coleoptera: Melolonthidae: Rutelinae y Dynastinae) con observaciones de su biología. Acta Zoológica Mexicana (n.s.) 20(3): 67-82.

Ramírez-Salinas, C., C. Pacheco-Flores y A.E. Castro-Ramírez. 2006. Cryptomeigenia sp. (Diptera: Tachinidae) como parasitoide de adultos de Phyllophaga (Phytalus) rufotestacea (Moser, 1918) (Coleoptera: Melolonthidae) en Chiapas, México. Acta Zoológica Mexicana (n.s.) 22(1): 1-8.

Ritcher, P.O. 1958. Biology of Scarabaeidae. Annual Review of Entomology 3: 311-334.

Ritcher, P.O. 1966. White grubs and their allies. A study of North American Scarabaeoid larvae. Studies in Entomology No.4, Oregon State University Press, Corvallis. 